\title{
Symmetrized Method with Optimized Second-Order Conditions for the Helmholtz Equation
}

\author{
Philippe Chevalier and Frédéric Nataf
}

\section{Introduction}

A schwarz type domain decomposition method for the Helmholtz equation is considered. The interface conditions involve second order tangential derivatives which are optimized (OO2, Optimized Order 2) for a fast convergence. The substructured form of the algorithm is symmetrized so that the symmetric-QMR algorithm can be used as an accelerator of the convergence. Numerical results are shown.

We consider the following type of problem: Find $u$ such that

$$
\begin{gathered}
\mathcal{L}(u)=f \text { in } \Omega \\
\mathcal{C}(u)=g \text { on } \partial \Omega
\end{gathered}
$$

where $\mathcal{L}$ and $\mathcal{C}$ are partial differential operators. We consider Schwarz-type methods for the solving of this problem. The original Schwarz algorithm is based on a decomposition of the domain $\Omega$ into overlapping subdomains and the solving of Dirichlet boundary value problems in the subdomains. It has been proposed in [15] to use of more general boundary conditions for the subproblems in order to use a nonoverlapping decomposition of the domain. The convergence speed is also increased dramatically.

More precisely, the computational domain $\Omega$ is decomposed into $N$ nonoverlapping subdomains:

$$
\bar{\Omega}=\bigcup_{i=1}^{N} \bar{\Omega}_{i}
$$

Let $\left(\mathcal{B}_{i j}\right)_{1 \leq i, j \leq N}$ be transmission conditions on the interfaces between the subdomains (e.g. Robin BC). What we shall call here a Schwarz type method for the problem (1) is its reformulation: Find $\left(u_{i}\right)_{1 \leq i \leq N}$ such that

$$
\begin{array}{r}
\mathcal{L}\left(u_{i}\right)=f \text { in } \Omega_{i} \\
\mathcal{C}\left(u_{i}\right)=g \text { on } \partial \Omega_{i} \cap \partial \Omega \\
\mathcal{B}_{i j}\left(u_{i}\right)=\mathcal{B}_{i j}\left(u_{j}\right) \text { on } \partial \Omega_{i} \cap \partial \Omega_{j}
\end{array}
$$

1991 Mathematics Subject Classification. Primary 65N55; Secondary 35J05. 
The above coupled system may be solved iteratively by a Jacobi algorithm:

$$
\begin{array}{r}
\mathcal{L}\left(u_{i}^{n+1}\right)=f \text { in } \Omega_{i} \\
\mathcal{C}\left(u_{i}^{n+1}\right)=g \text { on } \partial \Omega_{i} \cap \partial \Omega \\
\mathcal{B}_{i j}\left(u_{i}^{n+1}\right)=\mathcal{B}_{i j}\left(u_{j}^{n}\right) \text { on } \partial \Omega_{i} \cap \partial \Omega_{j}
\end{array}
$$

It is also possible, and indeed preferable, to write the resulting linear system in the interface unknowns $H=\left(\mathcal{B}_{i j}\left(u_{i}\right)\right)_{1 \leq i, j \leq N}$ (see [18])

$$
\mathcal{A} H=G
$$

( $G$ is some computable right hand side) and to solve it by conjugate gradient type methods.

Let us focus first on the interface conditions $\mathcal{B}_{i j}$. The convergence speed of Schwarz-type domain decomposition methods is very sensitive to the choice of these transmission conditions. The use of exact artificial (also called absorbing boundary conditions) boundary conditions as interface conditions leads to an optimal number of iterations, see $[\mathbf{1 2}, \mathbf{1 8}, \mathbf{1 1}]$. Indeed, for a domain decomposed into $N$ strips, the number of iterations is $N$, see [18]. Nevertheless, this approach has some drawbacks:

1. the explicit form of these boundary conditions is known only for constant coefficient operators and simple geometries.

2. These boundary conditions are pseudodifferential. The cost per iteration is high since the corresponding discretization matrix is not sparse for the unknowns on the boundaries of the subdomains.

For this reason, it is usually preferred to use partial differential approximations to the exact absorbing boundary conditions. This approximation problem is classical in the field of computation on unbounded domains since the seminal paper of Engquist and Majda [6]. The approximations correspond to "low frequency" approximations of the exact absorbing boundary conditions. In domain decomposition methods, many authors have used them for wave propagation problems $[3,5,14,4,2,16,1,19]$ and in fluid dynamics $[17,21,10,9]$. Instead of using "low frequency" approximations to the exact absorbing boundary conditions, it has been proposed to design approximations which minimize the convergence rate of the algorithm, see [22]. These approximations are quite different from the "low frequency" approximations and increase dramatically the convergence speed of the method, see $[\mathbf{1 3}]$ for a convection-diffusion equation. But, in the case of the Helmholtz equation, the same optimization procedure cannot be done, see Sec. 4 below. This is related to the existence of both propagative and evanescent modes for the solution of the Helmholtz equation. Roughly speaking, we will choose the interface conditions in Sec. 4 so that the convergence rate is small for both modes. In a different manner, in [20] overlapping decompositions are considered. In the overlapping regions, the partial differential equation is modified smoothly.

Another important factor is the choice of the linear solver (CG, GMRES, BICG, QMR, etc ... ) used to solve the substructured linear system (9). For such methods, the positivity and symmetry of the linear system are important issues [7]. Since the Helmholtz operator is not positive, it is unlikely that $\mathcal{A}$ is positive (and indeed, it is not). But, the Helmholtz operator is symmetric while $\mathcal{A}$ is not. By a change of unknown on $H$, we shall rewrite (9) such as to obtain a symmetric formulation. It enables us to use the symmetric-QMR algorithm described in [8]. 


\section{Substructuring}

In this section, we write the explicit form of the substructured linear problem (9) for the problem below. This section is classical. We want to solve the Helmholtz equation: Find $u$ such that

$$
\begin{array}{r}
\left.\left(\Delta+\omega^{2}\right)(u)=f \text { in } \Omega=\right] 0, L_{x}[\times] 0, L_{y}[ \\
\left.\left(\frac{\partial}{\partial n}+i \omega\right)(u)=g \text { on }\{0\} \times\right] 0, L_{y}\left[\cup\left\{L_{x}\right\} \times\right] 0, L_{y}[ \\
u=0 \text { on }] 0, L_{x}[\times\{0\} \cup] 0, L_{x}\left[\times\left\{L_{y}\right\}\right.
\end{array}
$$

where $i^{2}=-1, \frac{\partial}{\partial n}$ is the outward normal derivative and $\omega$ is positive. The domain is decomposed into $N$ nonoverlapping vertical strips:

$$
\left.\Omega_{k}=\right] L_{k-1, k}, L_{k, k+1}[\times] 0, L_{y}[, 1 \leq k \leq N
$$

with $L_{0,1}=0<L_{1,2}<\ldots<L_{N, N+1}=L_{x}$. The transmission condition on the interfaces is of the form $\mathcal{B}_{k}=\frac{\partial}{\partial n_{k}}+i \omega-\eta \frac{\partial^{2}}{\partial \tau_{k}{ }^{2}}$ where $\frac{\partial}{\partial \tau_{k}}$ is the tangential derivative on the boundary of $\partial \Omega_{k}$ and $\eta \in \mathbb{C}$ will be chosen in the section 4.

Problem (10) is equivalent to: Find $\left(u_{k}\right)_{1 \leq k \leq N}$ such that

$$
\begin{array}{r}
\left(\Delta+\omega^{2}\right)\left(u_{k}\right)=f \text { in } \Omega_{k} \\
\mathcal{B}_{k}\left(u_{k}\right)=\mathcal{B}_{k}\left(u_{k-1}\right) \text { on } \partial \Omega_{k} \cap \partial \Omega_{k-1} \\
\mathcal{B}_{k}\left(u_{k}\right)=\mathcal{B}_{k}\left(u_{k+1}\right) \text { on } \partial \Omega_{k} \cap \partial \Omega_{k+1} \\
\left(\frac{\partial}{\partial n}+i \omega\right)(u)=g \text { on }(\{0\} \times] 0, L_{y}\left[\cup\left\{L_{x}\right\} \times\right] 0, L_{y}[) \cap \partial \Omega_{k} \\
u=0 \text { on }(] 0, L_{x}[\times\{0\} \cup] 0, L_{x}\left[\times\left\{L_{y}\right\}\right) \cap \partial \Omega_{k}
\end{array}
$$

The continuity of the solution and its derivative on $\Omega_{k} \cap \partial \Omega_{k+1}$ are ensured by the interface conditions $\mathcal{B}_{k}$ and $\mathcal{B}_{k+1}$. Let $\Sigma_{k, k+1}=\partial \Omega_{k} \cap \partial \Omega_{k+1}, 1 \leq k \leq N$. Let us define now the vector of unknowns

$$
H=\left(\mathcal{B}_{1}\left(u_{1}\right)_{\mid \Sigma_{1,2}}, \ldots, \mathcal{B}_{k}\left(u_{k}\right)_{\mid \Sigma_{k-1, k}}, \mathcal{B}_{k}\left(u_{k}\right)_{\mid \Sigma_{k, k+1}}, \ldots, \mathcal{B}_{N}\left(u_{N}\right)_{\mid \Sigma_{N-1, N}}\right)
$$

Let $\Pi$ be the interchange operator on the interfaces, on each interface we have:

$$
\Pi\left(0, \ldots, 0, h_{k}^{k-1}, h_{k}^{k+1}, 0, \ldots, 0\right)=\left(0, \ldots, 0, h_{k-1}^{k}, h_{k+1}^{k}, 0, \ldots, 0\right)
$$

Let $T^{\prime}$ be the linear operator defined by:

$$
\begin{array}{r}
T^{\prime}\left(h_{1}^{2}, \ldots, h_{k}^{k-1}, h_{k}^{k+1}, \ldots, h_{N}^{N-1}, f, g\right)= \\
\left(\tilde{\mathcal{B}}_{1}\left(v_{1}\right)_{\mid \Sigma_{1,2}}, \ldots, \tilde{\mathcal{B}}_{k}\left(v_{k}\right)_{\mid \Sigma_{k-1, k}}, \tilde{\mathcal{B}}_{k}\left(v_{k}\right)_{\mid \Sigma_{k, k+1}}, \ldots, \tilde{\mathcal{B}}_{N}\left(v_{N}\right)_{\mid \Sigma_{N-1, N}}\right)
\end{array}
$$

where $\tilde{\mathcal{B}}_{k}=-\frac{\partial}{\partial n_{k}}+i \omega-\eta \frac{\partial^{2}}{\partial \tau_{k}{ }^{2}}$ and $v_{k}$ satisfies:

$$
\begin{array}{r}
\left(\Delta+\omega^{2}\right)\left(v_{k}\right)=f \text { in } \Omega_{k} \\
\mathcal{B}_{k}\left(v_{k}\right)=h_{k}^{k-1} \text { on } \partial \Omega_{k} \cap \partial \Omega_{k-1} \\
\mathcal{B}_{k}\left(v_{k}\right)=h_{k}^{k+1} \text { on } \partial \Omega_{k} \cap \partial \Omega_{k+1} \\
\left(\frac{\partial}{\partial n}+i \omega\right)\left(v_{k}\right)=g \text { on }(\{0\} \times] 0, L_{y}\left[\cup\left\{L_{x}\right\} \times\right] 0, L_{y}[) \cap \partial \Omega_{k} \\
v_{k}=0 \text { on }(] 0, L_{x}[\times\{0\} \cup] 0, L_{x}\left[\times\left\{L_{y}\right\}\right) \cap \partial \Omega_{k}
\end{array}
$$

It can be shown that 
LEMMA 1. Finding $\left(u_{k}\right)_{1 \leq k \leq N}$ solution to (11) is equivalent to finding $H=$ $\left(h_{1}^{2}, \ldots, h_{k}^{k-1}, h_{k}^{k+1}, \ldots, h_{N}^{N-1}\right)$ such that

$$
(I d-\Pi T)(H)=\Pi T^{\prime}(0,0, \ldots, 0, f, g) \equiv G
$$

where

$$
T=T^{\prime}(., ., \ldots, ., 0,0)
$$

\section{Symmetrization of the substructured system}

The linear system (18) may be solved by a relaxed Jacobi algorithm

$$
H^{n+1}=\theta \Pi T\left(H^{n}\right)+(1-\theta) H^{n}+G, \quad \theta \in(0,2)
$$

It is more efficient to use linear solvers based on Krylov subspaces such as GMRES, BICGSTAB or QMR. As mentioned in the introduction, the convergence rate of such methods is very sensitive to the positivity and symmetry of the linear system. Due to the non-positivity of the Helmholtz operator, it is unlikely that the substructured problem (18) be positive. Nevertheless, the Helmholtz equation is symmetric. It seems thus possible to obtain a symmetric reformulation of (18). For this, we need

DEFINITION 2. Let

$$
W=\prod_{k=2}^{N-1}\left(L^{2}(] 0, L_{y}[) \times L^{2}(] 0, L_{y}[)\right)
$$

be the space of complex valued traces on the interfaces. An element $H \in W$ is denoted

$$
H=\left(h_{1}^{2}, h_{2}^{1}, \ldots, h_{k}^{k-1}, h_{k}^{k+1}, \ldots, h_{N-1}^{N}, h_{N}^{N-1}\right)
$$

The space $W$ is endowed with a bilinear operator from $W \times W$ to $\mathbb{C}$

$$
\forall G, H \in W, \quad(G, H)_{b}=\sum_{k=2}^{N-1} \int_{0}^{L_{y}}\left(g_{k}^{k-1} h_{k}^{k-1}+g_{k}^{k+1} h_{k}^{k+1}\right)
$$

Let $A$ be a linear operator from $W$ to $W$. The transpose of $A$, denoted $A^{T}$ is the linear operator from $W$ to $W$ such that

$$
\forall G, H \in W, \quad(A G, H)_{b}=\left(G, A^{T} H\right)_{b}
$$

An operator $A$ is symmetric iff $A^{T}=A$.

The notion of symmetry for complex valued linear operator corresponds to the notion of complex symmetric linear systems studied in [8]. It is different from the notion of Hermitian operators since we have no conjugation. We have

Lemma 3. Let $\Pi$ be defined in (12). Then,

$$
\Pi^{T}=\Pi ; \Pi^{2}=I d
$$

and the operator $\Pi$ admits symmetric square roots. Let $\Pi^{1 / 2}$ denote one of these. Let $T$ be defined in (19). Then,

$$
T^{T}=T
$$


Proof. As for $\Pi$, it is enough to consider one interface. The operators $\Pi$ and $\Pi^{1 / 2}$ may be represented by the matrices

$$
\Pi=\left(\begin{array}{cc}
0 & 1 \\
1 & 0
\end{array}\right), \quad \Pi^{1 / 2}=\left(\begin{array}{cc}
1+\frac{i}{2} & 1-\frac{i}{2} \\
1-\frac{i}{2} & 1+\frac{i}{2}
\end{array}\right)
$$

As for $T$ the result comes from integration by parts of the Helmholtz operator.

This enables us to propose the following symmetrization:

Lemma 4. Problem (18) is equivalent to: Find $H \in W$ such that

$$
\left(I d-\Pi^{1 / 2} T \Pi^{1 / 2}\right)\left(\Pi^{-1 / 2} H\right)=\Pi^{-1 / 2} G
$$

Moreover

$$
\left(I d-\Pi^{1 / 2} T \Pi^{1 / 2}\right)^{T}=\left(I d-\Pi^{1 / 2} T \Pi^{1 / 2}\right)
$$

and the operators $\left(I d-\Pi^{1 / 2} T \Pi^{1 / 2}\right)$ and $(I d-\Pi T)$ have the same set of eigenvalues.

Other possibilities for symmetrizing (18) are

$$
(\Pi-T)(H)=\Pi(G) \text { or }(T-T \Pi T)(H)=T(G)
$$

They don't have the same eigenvalues as the original formulation and thus lead to slower convergence, see Figure 2.

\section{Optimization of the interface conditions}

The optimization is performed on the parameter $\eta$. The more $(I d-\Pi T)$ is close to the identity, the better the convergence of any iterative method will be. It seems then natural to minimize the norm of $\Pi T$. Since $\Pi$ is an isometry and does not depend on $\eta$, this is equivalent to minimize the norm of $T$. But we have

Proposition 5. Let the plane $\mathbb{R}^{2}$ be decomposed into two half-spaces $\Omega_{1}=$ ]$-\infty, 0\left[\times \mathbb{R}\right.$ and $\left.\Omega_{2}=\right] 0, \infty[\times \mathbb{R}$. For all $\eta \in \mathbb{C}$,

$$
\|T\|=1
$$

Indeed, a simple Fourier analysis shows that the operator $T(., ., 0,0)$ may be represented as an operator valued matrix:

$$
T=\left(\begin{array}{cc}
R & 0 \\
0 & R
\end{array}\right)
$$

where $R$ is a pseudo-differential operator whose symbol is

$$
\rho(k, \eta)=\frac{i \sqrt{\omega^{2}-k^{2}}-i \omega-\eta k^{2}}{i \sqrt{\omega^{2}-k^{2}}+i \omega+\eta k^{2}}
$$

where $k$ is the dual variable of $y$ for the Fourier transform. For $k=\omega, \rho(\omega, \eta)=1$ for all $\eta \in \mathbb{C}$. $[13]$.

It is thus impossible to optimize the spectral radius of $T(., ., 0,0)$ as is done in

Nevertheless, for any value of $\eta,|\rho(0, \eta)|=0$. This indicates that the convergence rate should be good for low frequencies as it is already the case if $\eta=0$, see [3]. It seems thus interesting to use $\eta$ for having a good convergence rate also for high frequencies. From (23), it is then enough to consider $\eta$ real. The choice of $\eta$ is 
TABLE 1. Influence of the number of subdomains

\begin{tabular}{||l|c|c|c|c|c|c|c||}
\hline Number of subdomains & $\mathbf{2}$ & $\mathbf{3}$ & $\mathbf{4}$ & $\mathbf{6}$ & $\mathbf{1 0}$ & $\mathbf{1 5}$ & $\mathbf{2 0}$ \\
\hline Relaxed Jacobi + Robin BC $=0.5$ & 50 & 62 & 75 & 130 & 216 & 406 & 832 \\
\hline QMR + Optimized BC & 10 & 18 & 24 & 34 & 59 & 92 & 126 \\
\hline
\end{tabular}

TABLE 2. Nbr of iterations vs. nbr of points per wavelength

\begin{tabular}{||l|c|c|c||}
\hline & $\mathbf{1 0}$ pts / lo & $\mathbf{2 0}$ pts / lo & $\mathbf{4 0}$ pts /lo \\
\hline Relaxed Jacobi + Robin BC $=0.5$ & 130 & 195 & 299 \\
\hline QMR + Optimized BC & 34 & 38 & 41 \\
\hline
\end{tabular}

TABLE 3. Influence of $\omega$

\begin{tabular}{||l|c|c|c||}
\hline & $\boldsymbol{\omega}=\mathbf{6}$ & $\boldsymbol{\omega}=\mathbf{2 0}$ & $\boldsymbol{\omega}=\mathbf{6 0}$ \\
\hline Relaxed Jacobi + Robin BC & 130 & 155 & 664 \\
\hline QMR + Optimized BC & 34 & 50 & 60 \\
\hline
\end{tabular}

done by minimizing the integral of $|\rho(k, \eta)|$ over the evanescent modes "admissible" on the computational grid:

$$
\min _{\eta \in \mathbb{R}} \int_{\omega}^{k_{\max }}|\rho(k, \eta)| d k
$$

where $k_{\max } \simeq 1 / h$ and $h$ is the typical mesh size. This is all the more important that if $\eta=0,|\rho(k, 0)|=1$ as soon as $|k|>\omega$.

\section{Numerical results}

The Helmholtz equation was discretized by a 5 -point finite difference scheme. Except for Table 3, the number of points per wavelength was between 20 and 25 . The problems in the subdomains were solved exactly by LU factorization. As for the substructured problem, we compared different iterative methods: relaxed Jacobi algorithm and the symmetric-QMR method. The symmetric-QMR algorithm was chosen since it is adapted to complex symmetric linear systems. We also tested various interface conditions:

$$
\begin{gathered}
\mathcal{B}=\partial_{n}+i \omega(\text { Robin BC) } \\
\mathcal{B}=\partial_{n}+i \omega+\frac{i}{2 \omega} \partial_{\tau}^{2}(\text { Order } 2 \text { BC }) \\
\mathcal{B}=\partial_{n}+i \omega-\eta \partial_{\tau}^{2}(\text { optimized real } \eta, \text { cf }(24)) \\
\mathcal{B}=\partial_{n}+i \omega+\left(\frac{i}{2 \omega}-\eta\right) \partial_{\tau}^{2}(\text { Order } 2+\text { optimized real } \eta, \eta \text { as above })
\end{gathered}
$$

The stopping criterion was on the maximum of the error between the converged numeric solution and the approximation generated by the algorithm, $\|e\|_{\infty}<10^{-5}$. Figure 1 ( $\omega=6 \Longleftrightarrow$ three wavelengths per subdomain), Table 1, Table 2 and Table 3 enable to compare the proposed method with a relaxed Jacobi algorithm.

In Figure 2, only the formulation of the substructured problem is varied while the interface conditions are the same. Table 4 shows the importance of the interface conditions. We have used Jacobi's algorithm with different interface conditions. 


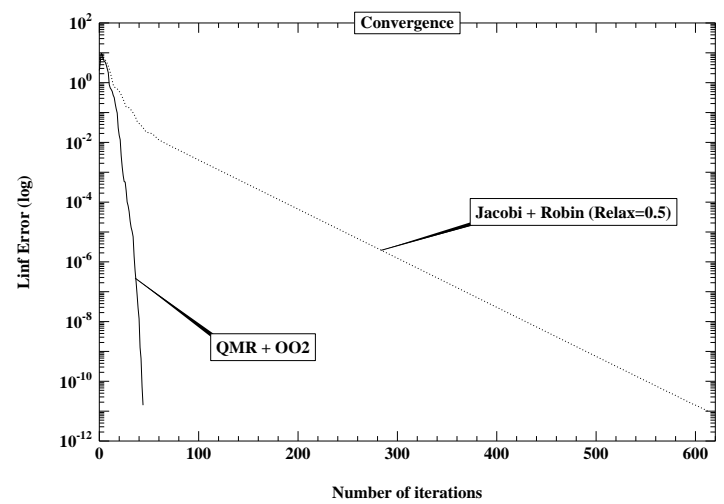

Figure 1. Comparison of the proposed method with a JacobiRobin algorithm

TABLE 4. Jacobi's algorithm - Influence of the interface conditions

\begin{tabular}{||l|c|c|c|c||}
\hline & Robin & Order 2 & $\eta$ real opt & $\begin{array}{c}\eta \text { real opt } \\
+ \text { order 2 }\end{array}$ \\
\hline Nbr of iterations & 195 & $>1000$ & 140 & 51 \\
\hline Best relaxation's coefficient & 0.5 & any & 0.6 & 0.9 \\
\hline
\end{tabular}

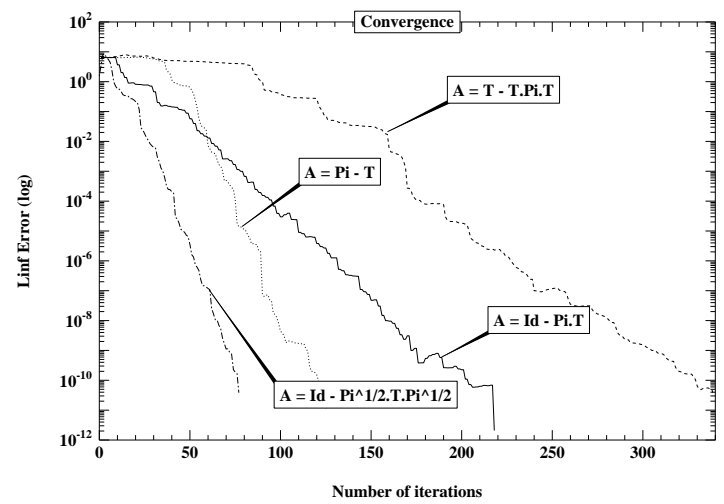

FigURE 2. Influence of the substructured formulation

\section{Perspectives}

We have presented a domain decomposition method for the Helmholtz equation and a decomposition of the domain into strips. The present method has already been extended to the Maxwell operator. We shall consider an arbitrary decomposition of the domain in a forthcoming paper.

\section{References}

1. A. de la Bourdonnaye, C. Fahrat, A. Macedo, F. Magoulès, and F.X. Roux, A nonoverlapping domain decomposition method for the exterior Helmholtz problem, DD10 Proceedings, 1997. 
2. M. Casarin, F. Elliot, and O. Windlund, An overlapping Schwarz algorithm for solving the Helmholtz equation, DD10 Proceedings, 1997.

3. B. Desprès, Domain decomposition method and the Helmholtz problem. II, Mathematical and numerical aspects of wave propagation. Proceedings of the 2nd international conference held (Kleinman Ralph eds. and al., eds.), SIAM, June 7-10 1993, pp. 197-206.

4. B. Desprès and J.D. Benamou, A domain decomposition method for the Helmholtz equation and related optimal control problems, J. Comp. Phys. 136 (1997), 68-82.

5. B. Despres, P. Joly, and J.E. Roberts, Domain decomposition method for the harmonic Maxwell equations, International Symposium on Iterative methods in linear algebra, 1991, pp. $475-484$.

6. B. Engquist and A. Majda, Absorbing boundary conditions for the numerical simulation of waves, Math. Comp. 31 (1977), no. 139, 629-651.

7. R.A Freund, G. Golub, and Nachtigal, Iterative solution of linear systems, Acta Numerica (1992), 57-100.

8. R.W. Freund, Conjuguate gradient-type methods for linear systems with complex symmetric coefficient matrices, J. Sci. Stat. Comput. 13 (1992), no. 1, 425-448.

9. M. Garbey, A Schwarz alternating procedure for singular perturbation problems, SIAM J. Sci. Comput. 17 (1996), no. 5, 1175-1201.

10. F. Gastaldi, L. Gastaldi, and A. Quarteroni, Adaptative domain decomposition methods for advection dominated equations, East-West J. Numer. Math. 4 (1996), no. 3, 165-206.

11. S. Ghanemi, P. Joly, and F. Collino, Domain decomposition method for harmonic wave equations, Third international conference on mathematical and numerical aspect of wave propagation, 1995, pp. 663-672.

12. T. Hagstrom, R.P. Tewarson, and A.Jazcilevich, Numerical experiments on a domain decomposition algorithm for nonlinear elliptic boundary value problems, Appl. Math. Lett. 1 (1988), no. 3, 299-302.

13. C. Japhet, Optimized Krylov-Ventcell method. application to convection-diffusion problems, DD9 Proceedings, John Wiley \& Sons Ltd, 1996.

14. B. Lichtenberg, B. Webb, D. Meade, and A.F. Peterson, Comparison of two-dimensional conformal local radiation boundary conditions, Electromagnetics 16 (1996), 359-384.

15. P.L. Lions, On the Schwarz alternating method III: A variant for nonoverlapping subdomains, Third International Symposium on Domain Decomposition Methods for Partial Differential Equations, SIAM, 1989, pp. 202-223.

16. L.C. McInnes, R.F. Susan-Resiga, D.E. Keyes, and H.M. Atassi, Additive Schwarz methods with nonreflecting boundary conditions for the parallel computation of Helmholtz problems, These proceedings.

17. F. Nataf and F. Rogier, Factorization of the convection-diffusion operator and the Schwarz algorithm, $\mathrm{M}^{3} \mathrm{AS} 5$ (1995), no. 1, 67-93.

18. F. Nataf, F. Rogier, and E. de Sturler, Domain decomposition methods for fluid dynamics, Navier-Stokes Equations and Related Nonlinear Analysis (A. Sequeira, ed.), Plenum Press Corporation, 1995, pp. 367-376.

19. B. Stupfel, A fast-domain decomposition method for the solution of electromagnetic scattering by large objects, IEEE Transactions on Antennas and Propagation 44 (1996), no. 10, 13751385.

20. Sun, Huosheng, Tang, and Wei-Pai, An overdetermined Schwarz alternating method, SIAM J. Sci. Comput. 17 (1996), no. 4, 884-905.

21. P. Le Tallec and T. Sassi, Domain decomposition with non matching grids, Tech. report, INRIA, 1991.

22. K.H. Tan and M.J.A. Borsboom, On generalized Schwarz coupling applied to advectiondominated problems, Domain decomposition methods in scientific and engineering computing. Proceedings of the 7th international conference on domain decomposition, vol. 180, AMS Contemp. Math., 1994, pp. 125-130.

Thomson-CSF Laboratoire Central de Recherche, 91404 Orsay Cedex, France

E-mail address: chevalie@thomson-lcr.fr - chevalie@cmapx.polytechnique.fr

C.M.A.P., CNRS UMR 7641, Ecole Polytechnique, 91129 Palaiseau Cedex, France

E-mail address: nataf@cmapx.polytechnique.fr 